\title{
Oscillation-based methods for actuation and manipulation of nano-objects
}

Cite as: AIP Conference Proceedings 1882, 020056 (2017); https://doi.org/10.1063/1.5001635 Published Online: 28 September 2017

V. L. Popov

\section{ARTICLES YOU MAY BE INTERESTED IN}

Drug loaded biodegradable load-bearing nanocomposites for damaged bone repair AIP Conference Proceedings 1882, 020025 (2017); https://doi.org/10.1063/1.5001604

Molecular level in silico studies for oncology. Direct models review AIP Conference Proceedings 1882, 020058 (2017); https://doi.org/10.1063/1.5001637

Two-dimensional Al hydroxide interaction with cancerous cell membrane building units: Complexed free energy and orientation analysis

AIP Conference Proceedings 1882, 020076 (2017); https://doi.org/10.1063/1.5001655
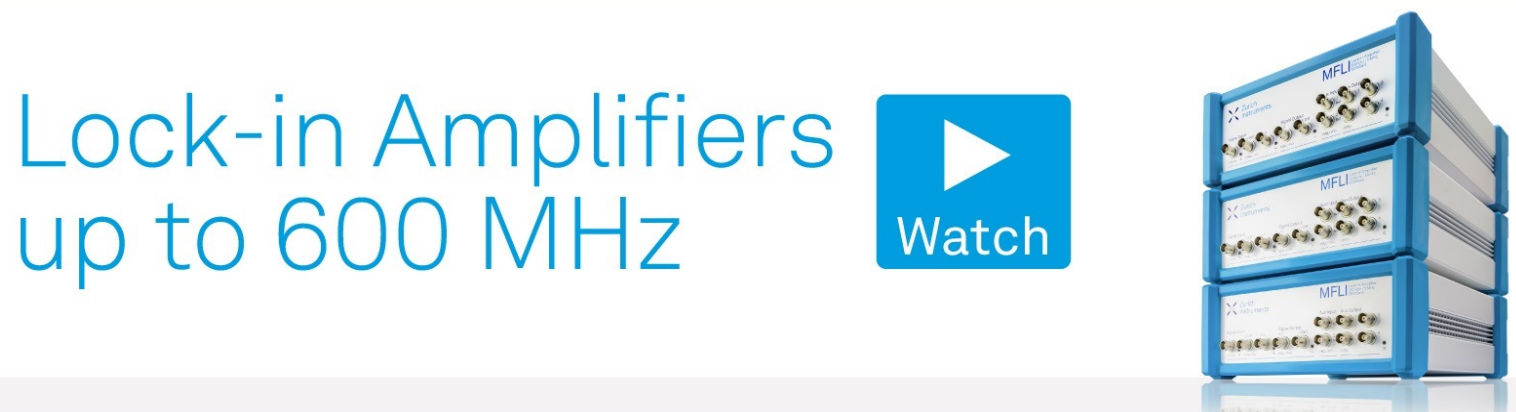


\title{
Oscillation-Based Methods for Actuation and Manipulation of Nano-Objects
}

\author{
V. L. Popov \\ Berlin University of Technology, Berlin, 10623 Germany \\ v.popov@tu-berlin.de
}

\begin{abstract}
We discuss how oscillations can be used for fixation or manipulation of nano-objects or producing nano-drives. The underlying principles are scale-invariant and principally can be scaled down up to the molecular scale. The main underlying principle of fixation and actuation occurs to be symmetry breaking of an oscillating system. From this unifying standpoint, a series of actuation principles are discussed as dragging, ratchets, micro walking, friction-inertia actuators, oscillation tweezers, flagella motors for propulsion in liquids as well as some recently proposed actuation principles.
\end{abstract}

\section{INTRODUCTION}

The tendency of miniaturization is one of the main global technological trends of engineering. However, the miniaturization in electronics and in mechanics proceeded with very different speeds. The reason for the very effective miniaturization in electronics, which lead to the contemporary world of computers, is that it was based just on the downscaling of transistor. The miniaturization of mechanical systems is not proceeding as rapid as in microelectronics, because many mechanical systems are not scalable. Let us look at one important part of our contemporary civilized world - a combustion engine. This engineering part is so important that it might be considered as a symbol of contemporary civilization. However, there exists no possibility to scale down this marvelous product of engineering to micro or even nano scale.

Of course, a simple way of just downscaling is very attractive, as it does not require any principally new solutions. And indeed, there were many attempts to produce very tiny machines of the same kind and based on the same principles as macroscopic engines. But regrettably, this way did not lead so far to much progress due to problems which such systems face on the microscale - high adhesion, high viscosity, if there are liquids in play, high wear-all these phenomena which can be summarized as "sticktion"-are the main obstacle of simple downscaling mechanical systems.

The same problem of scalability arises in systems designed for fixation and manipulation of objects. While on the macroscale the principles of a grip, a hand or of a claw arm are used in a variety of systems as tweezers or chop sticks, the principle is down scalable only to some extent.

It is therefore of interest to look for other driving principles which can be used at arbitrary scale. We will discuss here that in most cases, these are oscillation-based methods. The ingenious inventor Nikola Tesla said once: "If you want to find the secrets of the universe, think in terms of energy, frequency and vibration".

And this is true on the microscale even more than on the macroscale.

\section{OSCILLATION BASED DRIVES AND ACTUATORS}

\section{Ratchet}

One of the oscillation based driving mechanisms is used in mechanical watches. This is the famous "Ratchet and Pawl mechanism". It is based on an interaction of an oscillating unity with an asymmetrical substrate. 


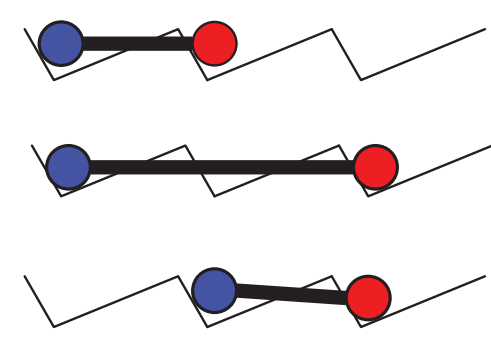

(a)

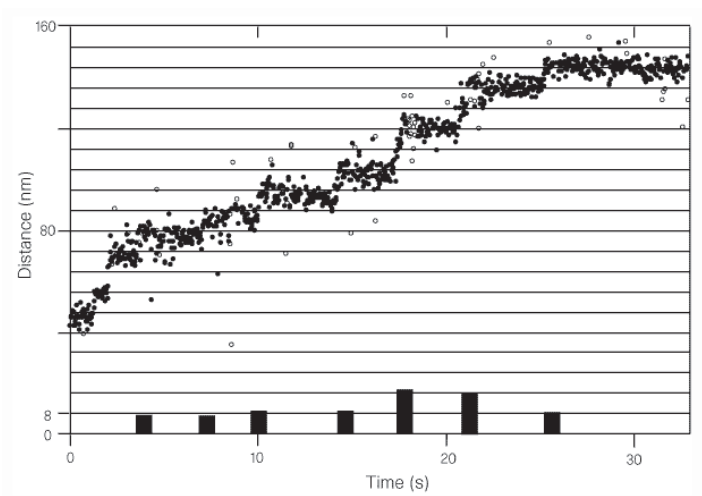

(b)

FIGURE 1. (a) Ratchet principles and movement of motor proteins. In (b) movement of a single molecule of kinesin along a micro-tubuli is shown (source: [1])

This mechanism can be realized both in rotational and translational version. If you have an asymmetrical substrate or asymmetrical potential relief and a two-body system capable of changing the length of the bond between the bodies, you will have a ratchet a pawl system. By both expanding and contracting of the bond the system moves in the same direction and after one cycle of oscillation is displaced by one period of the substrate. This would not work if the substrate were symmetrical. All such systems based on oscillation of a two-body system on an asymmetrical substrate are called "ratchets".

The ratchet principle is easily down scalable and is widely used by the living nature on the molecular scale in the form of the so-called motor proteins. The transport of motor proteins occurs along the so-called microtubule-fibers composing a sort of a skeleton of a cell. Microtubuli have periodic structure which is asymmetric with respect of two ends: thus if one would stroke a tubula, one would have different feeling by stroking forth and back. The transport units are the so-called motor proteins. A typical motor protein consists of two heads connected with a high molecular bond. Depending on temperature, the bond can undergo transition from a gas-like coiled to a liquid-like globular state. In this way, the length of the bond can be changed. The transport proceeds due to the "ratchet and pawl" principle: due to asymmetry of the substrate, both expansion and contraction of the molecule are going in one preferred direction, so that after one cycle the molecule is shifted by one period of the structure of the microtubule (Fig. 1a). In Fig. 1b experimental observation of the position of one molecule of kinesin is presented [1], which shows that the molecule moves in steps, and the length of one step is exactly equal to the period of the structure of the microtubula. The expansion and contraction of the motor protein occurs due to consumption of one ATPmolecule. For each step is needed exactly one ATP molecule.

Ratchets are, however not the only oscillation based principle which can be easily downscaled. The other one principle is the "Walking".

\section{Walking}

This principle means moving one leg forth when the normal force acting on it is small. This can be done either by lifting one of the legs in each step or just by reducing the normal force as shown in Fig. 2 . The same can be realized on the structured substrates.

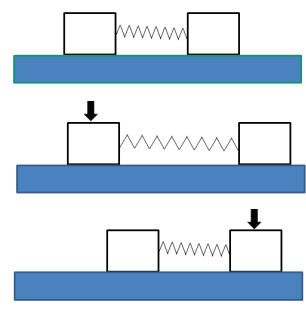

(a)

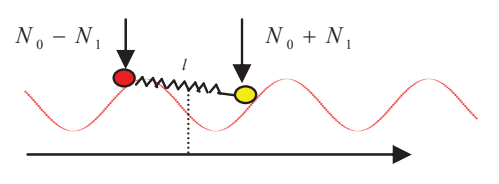

(b)

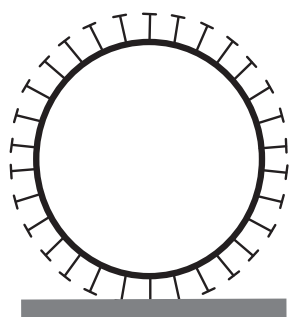

(c)

FIGURE 2. (a) Walking on a substrate with friction, (b) walking on a structured substrate, (c) roll movement is based on the same basic principle as walking 

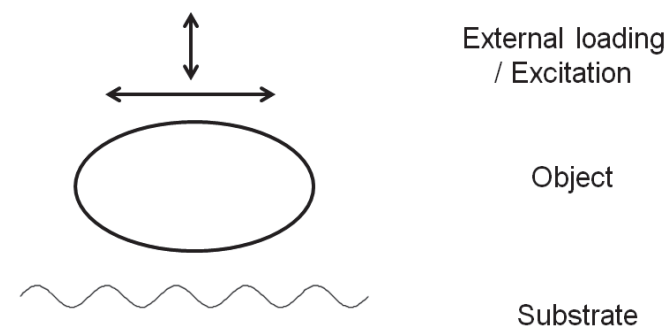

FIGURE 3. General scheme of an object interacting with a substrate and subjected to a vibrational load. If both the substrate and load are symmetric, no macroscopic movement is generated

In $[2,3]$ it was shown that when the normal force and the binding length of a two-body machine placed in a periodic potential are changing in such a way they circle around some points in the parameter space, this will lead to directed motion of the system. The directed motion is even possible when there is force acting opposite to the direction of motion. The principle of walking can be generalized for the case of many legs (caterpillar), infinite many legs (traveling wave motor) or only one leg (jumping). An interesting recent analysis of actuator types which can be achieved with only on "leg" by applying to it a superposition of normal and tangential oscillations can be found in [4]. It is interesting to note that rolling is based on the same basic principle as walking and can be seen as "caterpillar-like walking with periodic boundary conditions (Fig. 2c).

\section{SYMMETRY BREAKING AS A GENERAL PRINCIPLE OF PROPULSION}

The discussed two propulsion principles as well as many others have many common features. First to manipulate an object, one has to have an object which has to be manipulated. Then, if we speak about transport on solid substrate, there should be a substrate, and finally the object is subjected to oscillating external load which may be a periodic force or periodic change of a bond length. If all parts of the system are symmetrical, then we will have only oscillations but no net directed movement (Fig. 3).

For inducing directed movement, the symmetry of the system has to be broken. There are many possibilities to do this. Each kind of broken symmetry will produce a class of possible drives (Fig. 4).

The simplest idea is just to apply an external force (Fig. 4a). This is a very old kind of breaking the symmetry known as dragging or pulling or pushing. Already on this example one can see two features which will be common for all other types of drives: The breaking the symmetry and the existence of a threshold. For inducing a directed motion, a threshold force has to be applied (the static force of friction). This "threshold principle" does exist also in most of other oscillation based drives. The principle of dragging is used for example by manipulation of nanoobjects by a tip of atomic force microscope [5].

Let us now take the force away and instead introduce an asymmetry of the substrate. We get a ratchet which was already discussed above Fig. 4b. Ratchets also need both an asymmetry and some threshold amplitude of asymmetry to initiate a movement.

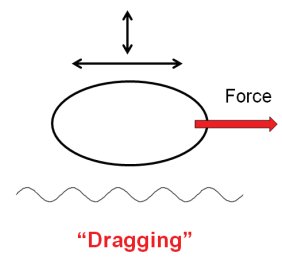

(a)

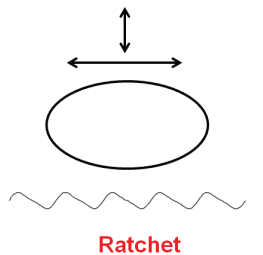

(b)

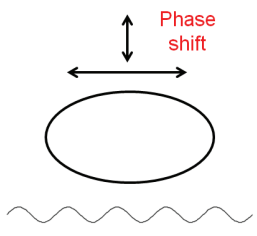

Walking or Jumping

(c)

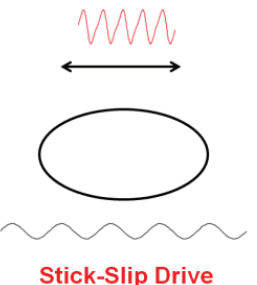

(d)

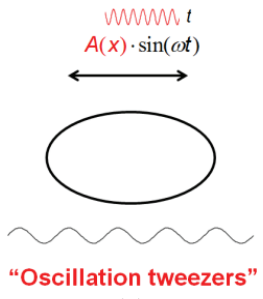

(e)

FIGURE 4. Possible types of symmetry breaking: application of force (dragging), using asymmetrical substrate (ratchet), introducing asymmetry by a phase shift of normal and tangential oscillations (walking), introducing an asymmerty of the driving signal (stick-slip-drive) or introducing the coordinate dependency of the oscillation amplitude ("oscillation tweezers") 


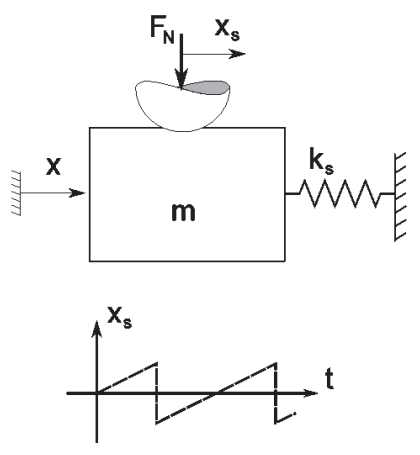

(a)

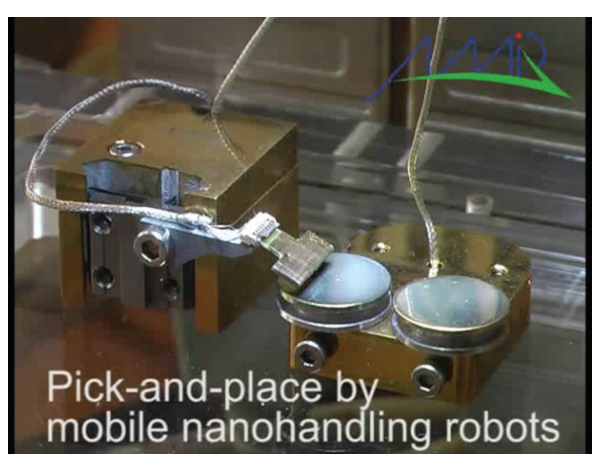

(b)

FIGURE 5. (a) A scheme of a stick-slip drive and (b) a robot based on stick-slip drives (S. Fatikow, Uni Oldenburg, see [6])

If we now take the asymmetry of the substrate away and instead break the symmetry by introducing a phase shift between the normal and tangential oscillations, we get the principle of walking or, if there is only one leg, of jumping. An advantage of walking is that one can easily reverse the direction by changing the phase shift. This "walking" principle is a basis of many automated transport systems and feeders (as e.g. vibrational trays).

Till now we considered a symmetrical excitation with a sin- or cos-law. The symmetry can be broken also at this point producing the so-called stick-slip-drives (Fig. 4d) [6]. The principle of a stick-slip-drive is shown in more detail in Fig. 5a, while Fig. 5b shows a micro handling robot using several stick-slip drives for actuation (S. Fatikow, University of Oldenburg).

Finally, one can introduce a dependence of the amplitude of oscillations on coordinate e.g. amplitude of the laser light) which, depending on the system, leads to directed drift either in the direction of the minimum [7] or maximum of oscillation amplitude $[8,9]$.

\section{PROPULSION IN LIQUIDS}

People and fishes normally use for propulsion in liquids either the existence of a free surface or turbulent flows creating a pressure difference between a laminar part of flow and the "shadow" in the turbulent part. At the nanoscale, the Reynolds number is very small, so the flows are laminar and it is as difficult to create propulsion as to swim in a bog. The problem is that any reciprocal movement creates an exactly zero net momentum, thus the "swimming" is only possible in the direction of the force of gravity. However, a wave like relative movement of at least three bodies was shown to lead to propulsion even in the linearly viscous liquid at low Reynolds numbers [10] (Fig. 7a). The same effect can be achieved even simpler by rotating a spiral which also produces a wave propagating from one end of spiral to the other (Fig. 7b). According to paleontological data, this principle of rotating spiral is at the same time one of the oldest principles of propulsion used by nature: a flagella motor [11]. It consists of one single fiber bent in a form of a spiral. Its rotation caused by an ion flow through the cell membrane leads to directed motion of the cell [5]. At rotational frequency of about $100 \mathrm{~Hz}$, bacteria achieve velocities of the order of 10$20 \mu \mathrm{m} / \mathrm{s}$. This principle can be used for creation of micro-robots [12].

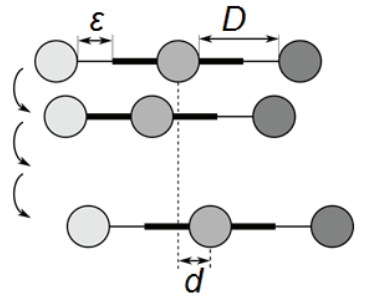

(a)

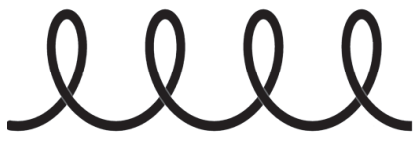

(b)

FIGURE 6. Propulsion in liquids at low Reynolds numbers: (a) a wavy relative movement of at least three bodies, (b) rotation of a "flagella" 


\section{CONCLUSIONS}

Using the concept of symmetry breaking, we discussed the general concept for nanoscale actuators based on symmetry breaking of an oscillating system. The suggested principle of symmetry breaking is scale invariant. It covers many known types of actuators and can be used for designing or optimization of new types of micro- and nano-handling devices as e.g. recently suggested oscillation rolling drive [13]. The ultimate goal would be creation of autonomous micro-robots which can be used for multiple purposes in medicine, e.g. for drug delivery [14, 15]. A further interesting topic would be analyzing the function of biologically active molecules (enzymes) and surfaces (membranes) from the point of view of mechanical actuation $[16,17]$.

\section{ACKNOWLEDGMENTS}

The author acknowledges valuable discussions with R. Wetter.

\section{REFERENCES}

1. W. Hua, E. C. Young, M. L. Fleming, and J. Gelles, Nature 388, 390-393 (1997).

2. V. L. Popov, Phys. Rev. E 68, 026608 (2003).

3. V. L. Popov, Int. J. Non-Linear Mechanics 39(4), 619-633 (2003).

4. M. Popov and Q. Li, arXiv: 1705.03379 (2017).

5. P. Nita, S. Casado, D. Dietzel, A. Schirmeisen, and E. Gnecco, Nanotechnology 24, 325302 (2013).

6. H. X. Nguyen, E. Teidelt, V. L. Popov, and S. Fatikow, Arch. Appl. Mech. 86, 1771-1785 (2016).

7. P. L. Kapitza, Sov. Phys. JETP-USSR 21, 588-597 (1951).

8. A. Ashkin, History of Optical Trapping and Manipulation of Small Neutral Particles, Atoms, and Molecules, in Single Molecule Spectroscopy (Springer, Berlin, 2001), pp. 1-24.

9. J. R. Moffitt, Y. R. Chemla, S. B. Smith, and C. Bustamante, Annu. Rev. Biochem. 77, 205-228 (2008).

10. A. Najafi and R. Golestanian, Phys. Rev. E 69, 062901 (2004).

11. H. C. Berg and R. A. Anderson, Nature 245, 380-382 (1973).

12. V. Magdanz, S. Sanchez, and O. G. Schmidt, Adv. Mater. 25, 6581 (2013).

13. R. Wetter and V. L. Popov, Phys. Mesomech. 19, 167-172 (2016).

14. S. Martel, J. Micro-Bio Robotics 8, 41-52 (2013).

15. R. Mhanna et al., Small 10, 1953-1957 (2014).

16. A. Tsukanov and S. Psakhie, Adv. Biomater. Devices Med. 2, 44-53 (2015).

17. A. Tsukanov and S. Psakhie, Facta Univer. Mech. Eng. 14(3), 269-280 (2016). 